\title{
Übersichten
}

Somnologie $2021 \cdot 25: 38-44$

https://doi.org/10.1007/s11818-020-00271-8

Eingegangen: 10. Juli 2020

Angenommen: 3. Oktober 2020

Online publiziert: 22 . Oktober 2020

(c) Der/die Autor(en) 2020

\author{
Victoria Vaas ${ }^{1}(\mathbb{D}) \cdot$ Christoph Fisser $^{1} \cdot$ Maria Tafelmeier $^{1} \cdot$ Dominik Linz $^{2} \cdot$ \\ Michael Arzt ${ }^{1}$ \\ 'Schlafmedizinisches Zentrum der Klinik und Poliklinik für Innere Medizin II, Universitätsklinikum \\ Regensburg, Regensburg, Deutschland \\ ${ }^{2}$ Department of Cardiology, Maastricht University Medical Centre and Cardiovascular Research Institute \\ Maastricht, Maastricht, Niederlande
}

\section{Interaktionen schlafbezogener Atmungsstörungen mit Vorhofflimmern}

\section{Pathophysiologie und Klinik}

Vorhofflimmern (VHF) ist die häufigste Herzrhythmusstörung. Aufgrund der sich ändernden Altersstruktur in den westlichen Ländern und der Zunahme von Risikofaktoren wie Bluthochdruck und Übergewicht steigt die Prävalenz der Patienten mit VHF $[1,2]$. Aufgrund der häufigen Herzfrequenzanstiege trägt VHF zu einer Tachymyopathie und einem erhöhten Risiko für Schlaganfälle (20-30\% aller Schlaganfälle) bei [2-5]. Sogar bei Patienten mit einer effektiven Rhythmuskontrolle durch eine leitliniengerechte Behandlung ist die Mortalitätsrate durch Pumpversagen sowie plötzlichen Herztod erhöht [6]. Die Volkskrankheit VHF stellt auch eine erhebliche sozioökonomische Belastung dar [2]. Die durch VHF verursachten jährlichen Kosten werden im Jahr 2017 in Deutschland auf 660 Mio. $€$ geschätzt [7]. Bei der Therapie von VHF hat die Modifikation von Risikofaktoren eine wichtige Bedeutung [8]. $\mathrm{Zu}$ den Risikofaktoren für VHF zählen unter anderem Übergewicht, Bluthochdruck, Diabetes mellitus und die schlafbezogene Atmungsstörung (SBAS) [8]. Aufgrund der erhöhten Prävalenz der SBAS (21-74\%) bei VHFPatienten verglichen mit einer Kontrollgruppe spielt diese eine wichtige Rolle im Zusammenhang mit VHF [9-14]. Dieser Wert variiert aufgrund der verschiedenen Diagnose- und Schweregradkriterien in den Studien [9-13]. Sowohl die obstruktive Schlafapnoe (OSA) als auch die zentrale Schlafapnoe (ZSA) sind mit VHF assoziiert [15]. Die zugrunde liegenden Mechanismen sind der oxidative Stress, die Aktivierung des sympathischen Nervensystems, die Erhöhung der atrialen und ventrikulären transmuralen Wandspannung sowie strukturelles und elektrophysiologisches Remodeling der Vorhöfe ([8, 16]; • Abb. 1).

Bei Patienten mit unbehandelter OSA sind die VHF-Rezidivraten nach elektrischer Kardioversion und Vorhofflimmerablation (Pulmonalvenenisolation) um $31 \%$ erhöht [17]. Trotz der bisherigen Erkenntnisse bleibt aufgrund fehlender konfirmatorischer randomisierter Studien unklar, wie sich eine Behandlung der Schlafapnoe auf die Rezidivhäufigkeit von VHF nach elektrischer Kardioversion und Pulmonalvenenisolation auswirkt. Deshalb stellt dieser Übersichtsartikel die epidemiologischen und pathophysiologischen Zusammenhänge zwischen SBAS und VHF dar. Auf die Unterschiede zwischen der OSA und der ZSA wird eingegangen. Der Artikel fasst die Ergebnisse der wichtigsten Behandlungsstudien und Therapieempfehlungen zusammen. Ein sich daraus ergebender Forschungsbedarf wird aufgezeigt.

\section{Pathophysiologie \\ Akute transiente arrhythmogene Mechanismen}

\section{Intermittierende Hypoxie}

Obstruktive Apnoen und Hypopnoen führen $\mathrm{zu}$ intermittierenden Sauerstoffentsättigungen. Es entstehen freie Sauerstoffradikale („reactive oxygen species“, ROS) und die sympathische und parasympathische Aktivität wird erhöht ([18, 19]; - Abb. 1). Dieses Phänomen tritt durch Inhibierung des mitochondrialen Elektronentransportes, erhöhte Aktivität reduzierter NADPHs und Xanthinoxidasen sowie erniedrigte Spiegel von Antioxidantien auf [18, 20, 21]. In Tiermodellen führte intermittierende Hypoxie ROS-vermittelt zu Hypertrophie, Längenzunahme von Myokardzellen sowie $\mathrm{zu}$ erhöhten Apoptoseraten $[22,23]$. In einer randomisierten kontrollierten Studie konnte nachgewiesen werden, dass bei Patienten mit OSA durch eine „continuous positive airway pressure“ (CPAP) Therapiemarker oxidativen Stresses (z. B. 8-Isoprostane) relevant reduziert werden [24]. Hypoxämie löst unter physiologischen Bedingungen durch das Einwirken auf die Glomera carotica Hyperventilation und eine Aktivierung des Sympathikus aus [19, 25]. Im Vergleich dazu führt intermittierende Hypoxie bei Obstruktion der oberen Atemwege durch das Verhindern 


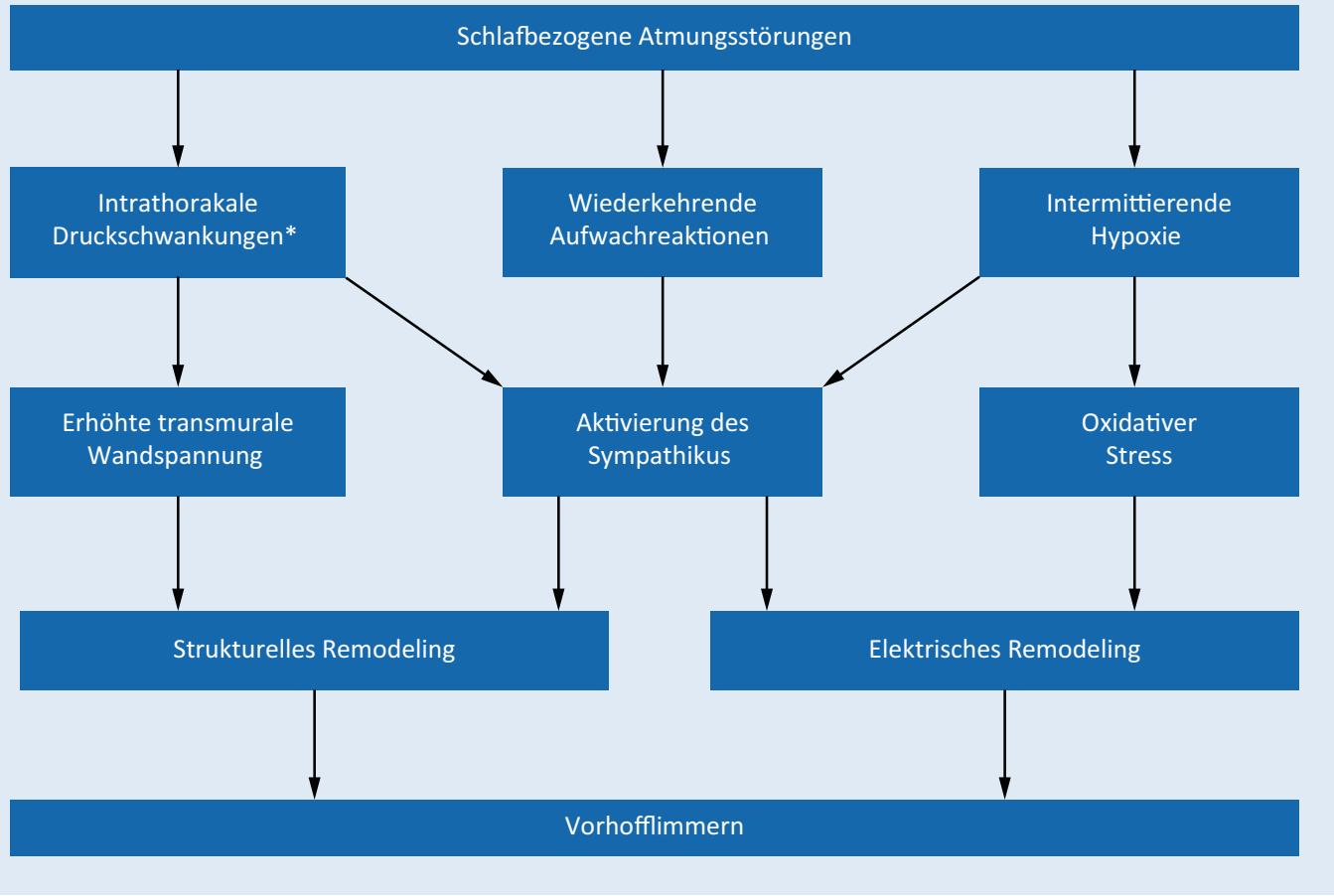

Abb. $1 \varangle$ Hauptmechanismen für die Assoziation zwischen schlafbezogenen Atmungsstörungen und kardialen Arrhythmien. Asterisk nicht bei zentraler Schlafapnoe der Lungenexpansion sowie die Dehnung von vagolytischen Nervenfasern der Lunge zum Tauchreflex (Erhöhung der sympathischen Vasokonstriktion der Muskeln) [26]. Dies führt zur Steigerung des Blutdruckes und einer vagal induzierten Bradykardie [19, 25]. Folglich besteht die Reaktion des Körpers auf intermittierende Hypoxie aus der direkten und indirekten Aktivierung des Sympathikus sowie der Aktivierung des Parasympathikus [16]. Die Häufigkeit und Intensität der Sauerstoffentsättigungen korreliert mit dem Auftreten atrialer und ventrikulärer Arrhythmien [27-30]. Die ZSA ist weniger mit intermittierender Hypoxie und damit verbundenen Aktivierungen des parasympathischen und sympathischen Nervensystems assoziiert als die OSA [31].

\section{Kombinierte sympathovagale Aktivierung}

Sowohl Apnoen als auch Hypopnoen und mildere Flusslimitationen können in Kombination mit verstärkter Atemanstrengung zu respiratorisch bedingten Aufwachreaktionen führen. Diese können auch bei respiratorischen Ereignissen ohne Sauerstoffentsättigungen auftreten. Bei gesunden Testpersonen, bei denen durch akustische und mechanische
Stimuli Aufwachreaktionen provoziert wurden, stellte man einen signifikanten Anstieg der Herzfrequenzvariabilität und insbesondere ihrer sympathischen Komponente fest [32]. Die resultierende Hyperpnoe nach einer Aufwachreaktion dehnt die peripheren afferenten Fasern der Lunge, was mit einer vagolytischen Reaktion (Hering-BreuerReflex) assoziiert ist und zusammen mit erregungsbedingten Erhöhungen des Sympathikotonus die Herzfrequenz steigert [19, 25, 33, 34]. Des Weiteren zeigten Horner et al., dass spontane Aufwachreaktionen kombiniert mit einem akuten Anstieg der Herzfrequenz bei Hunden mithilfe von Blockaden des Sympathikus verhindert werden konnten [33].

Sowohl OSA- als auch ZSA-Patienten weisen im Vergleich zu Kontrollpatienten einen höheren Sympathikotonus auf ([35, 36]; siehe - Abb. 1). Dieser ist nicht nur während einer Schlafapnoe-Episode, sondern auch tagsüber erhöht $[35,36]$. Folgen sind eine erhöhte Herzfrequenz und ein erhöhter Blutdruck, ebenfalls mit ausgeprägteren Effekten während der Schlafphase [35-37].

\section{Intrathorakale Druckschwankungen}

Obstruktive Apnoen und Hypopnoen führen $\mathrm{zu}$ einem inspiratorischen Einsatz der Atemhilfsmuskulatur, um den kollabierten oberen Atemwegen entgegenzuwirken. Dadurch kann ein negativer intrathorakaler Druck von bis $\mathrm{zu}-80 \mathrm{mmHg}$ entstehen [16]. Beim Menschen ist der durch das MüllerManöver (simulierte obstruktive Apnoe) hervorgerufene negative intrathorakale Druck mit einem erheblichen Anstieg der postganglionären sympathischen Nervenaktivität um mehr als $200 \%$ verbunden. Dies führt zu einem signifikanten Anstieg des mittleren Blutdrucks am Ende der Apnoe [38]. Gleichzeitig führt ein negativer intrathorakaler Druck während ineffektiver Inspiration $\mathrm{zu}$ einer ausgeprägten, aber transienten vagalen Aktivierung, die mit einer deutlichen Verkürzung der atrialen Refraktärzeit und Aktionspotentialdauer als auch einer Zunahme der VHF-Induzierbarkeit vergesellschaftet ist [39]. Die Erhöhung der atrialen und ventrikulären Wandspannung bei Patienten mit OSA wird mit den Schwankungen der intrathorakalen Druckverhältnisse sowie dem nächtlichen Blutdruckanstieg assoziiert [16, 40, 41]. Durch erhöhte 
negative intrathorakale Druckverhältnisse werden der venöse Rückfluss zum Herzen und die atriale und ventrikuläre Vorlast erhöht [42, 43]. Simulierte OSA ist bei gesunden Versuchspersonen mit einer akuten Zunahme des Durchmessers der proximalen Aorta, des links ventrikulären sowie atrialen Volumens, gemessen durch Echokardiographie, sowie der Reduzierung der links ventrikulären Ejektionsfraktion (LVEF) assoziiert [44-46]. Bei Patienten mit Herzinsuffizienz und schwerer OSA sinkt der ösophageale Druck $\left(\mathrm{P}_{\mathrm{es}}\right)$ um $2 \mathrm{~mm} \mathrm{Hg}$ und der systolische Blutdruck steigt um $14 \mathrm{~mm} \mathrm{Hg}$ während des NonREM-Schlafes (Schlafstadium 2) [40]. Folglich nimmt die systolische links ventrikuläre transmurale Wandspannung $\left(\mathrm{LVP}_{\mathrm{tm}}=\right.$ arterieller Blutdruck + Betrag des negativen intrathorakalen Druckes) verglichen mit dem Wachzustand um $16 \mathrm{~mm} \mathrm{Hg} \mathrm{zu} \mathrm{[40].} \mathrm{Diese} \mathrm{Erhöhung} \mathrm{der}$ linksventrikulären Nachlast ist vor allem in Ventilationsphasen nach einer Apnoeperiode zu beobachten [40]. Durch eine Kombination der Blutdrucksenkung sowie der Erhöhung des $\mathrm{P}_{\mathrm{es}}$ durch eine CPAP-Therapie konnte die systolische $\mathrm{LVP}_{\mathrm{tm}}$ während des Schlafstadiums 2 um $20 \mathrm{mmHg}$ reduziert werden [40]. Dieses Phänomen kann auf die Vorhofwände übertragen werden, welche aufgrund der geringeren Wanddicke im Vergleich zum linken Ventrikel sensibler auf die Erhöhung der linksventrikulären transmuralen Wandspannung reagieren.

\section{Chronische arrhythmogene Mechanismen}

\section{Strukturelles Remodeling der Vorhöfe}

Repetitive obstruktive respiratorische Ereignisse können durch wiederholte mechanische Vorhofdehnung und häufige Episoden von Des- und Reoxygenierung strukturelles Remodeling sowie Myokardschäden verursachen [31, 42, $43,47]$. Zudem ist die zyklische Desund Reoxygenierung im Zusammenhang mit der Schlafapnoe vergleichbar mit einer Ischämie oder Reperfusionsschäden, die die Produktion reaktiver Sauerstoffspezies, das Auftreten von Gefäßentzündungen und den Blutdruck

Somnologie 2021 · 25:38-44 https://doi.org/10.1007/s11818-020-00271-8

(c) Der/die Autor(en) 2020

V. Vaas $\cdot$ C. Fisser $\cdot$ M. Tafelmeier $\cdot$ D. Linz $\cdot$ M. Arzt

\section{Interaktionen schlafbezogener Atmungsstörungen mit} Vorhofflimmern. Pathophysiologie und Klinik

\section{Zusammenfassung}

Hintergrund. Vorhofflimmern (VHF) ist die häufigste Herzrhythmusstörung. Sowohl die zentrale als auch die obstruktive Schlafapnoe interagieren mit dieser Erkrankung. Intermittierende Hypoxie, oxidativer Stress, wiederkehrende Aufwachreaktionen, intrathorakale Druckveränderungen und atriales Remodeling können im Rahmen einer schlafbezogenen Atmungsstörung (SBAS) zu VHF führen.

Ziel. Dieser Artikel stellt die komplexen Zusammenhänge und Erkenntnisse jüngster Forschungen bezüglich SBAS und VHF sowie die Therapiemöglichkeiten dar.

Material und Methoden. Es erfolgten eine Literaturrecherche von Original- und Übersichtsartikeln sowie Metaanalysen, die zwischen 1963 und 2020 in der PubMedDatenbank veröffentlicht wurden. Ergebnisse. Die Erkenntnisse der Studien weisen auf einen bidirektionalen kausalen Zusammenhang zwischen SBAS und VHF hin.
Die pathophysiologischen Auswirkungen der obstruktiven und zentralen Schlafapnoe auf VHF sind unterschiedlich. Die Studien, die die Effekte einer Therapie der SBAS auf das Rezidivrisiko von VHF nach Intervention (Kardioversion oder Pulmonalvenenisolation) untersuchen, ergeben bisher kein eindeutiges Bild.

Diskussion. Bisherige Studien bestätigen multiple Interaktionen zwischen SBAS und VHF. Aufgrund widersprüchlicher Ergebnisse hinsichtlich der Effekte einer positiven Atemwegsdrucktherapie auf das Rezidivrisiko von VHF nach Interventionen sind weitere Studien nötig.

Schlüsselwörter

Schlafbezogene Atmungsstörungen . Vorhofflimmern - Partiale Arrhythmien . Continuous positive airway pressure . Adaptive Servoventilation

\section{Interactions between sleep apnea and atrial fibrillation. Pathophysiology and symptoms}

\section{Abstract}

Background. Atrial fibrillation (AF) is the most common cardiac arrhythmia. Central as well as obstructive sleep apnea interact with this condition. Intermittent hypoxia, oxidative stress, repeated awakening, intrathoracic pressure swings, and atrial remodeling as part of sleep-disordered breathing (SDB) can lead to AF.

Objectives. This article focuses on the complex relationship and recent research findings concerning SDB and AF, as well as the therapeutic options.

Materials and Methods. A literature search was conducted of original and review articles, as well as meta-analyses, published in the PubMed database between 1963 and 2020. Results. The findings of the studies indicate a bidirectional relationship between SDB and
AF. The pathophysiological consequences of obstructive and central sleep apnea vary. The studies evaluating the effects SDB treatment on the recurrence of $A F$ after intervention (e.g., cardioversion and pulmonary vein isolation) do not yet provide a clear picture. Conclusions. Previous studies suggest multiple interactions between SDB and AF. Considering the conflicting results on the effects of positive airway pressure treatment on the risk of recurrence of $A F$ after intervention, further studies are needed.

\section{Keywords}

Sleep-disordered breathing · Atrial fibrillation . Partial arrhythmia - Continuous positive airway pressure $\cdot$ Adaptive servoventilation erhöhen [48]. All diese Faktoren können Myokardschäden verursachen [48]. Auf die relativ dünnen Wände der Vorhöfe wirken sich diese Effekte besonders aus [31, 47]. Intermittierende Hypoxie bei Ratten bewirkte nach vier Wochen simulierter Schlafapnoe Vorhofleitungs- anomalien, die mit einer ConnexinDysregulation und einer erhöhten Vorhoffibrose verbunden sind [49]. Weitere Studien stützen die Beobachtung von strukturellen Anomalien wie Vorhoffibrose, Herunterregulierung oder Lateralisierung von Connexin 43 und einer 


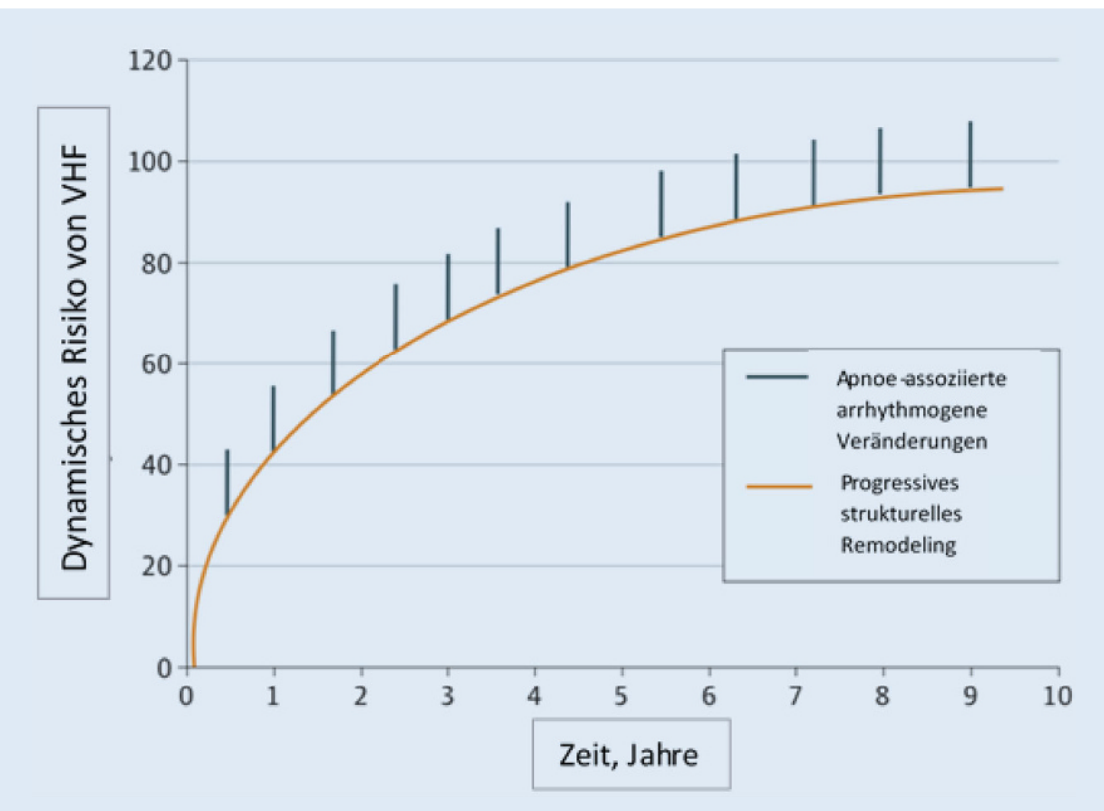

Abb. $2 \Delta$ Apnoeassoziierte arrhythmogene Veränderungen und progressives strukturelles Remodeling in Korrelation zum Risiko von Vorhofflimmern (VHF) [52]

erhöhten autonomen Nervendichte [49, 50]. Dementsprechend zeigten Patienten mit lang andauernder OSA deutliche atriale strukturelle Veränderungen [49]. Zusätzliche Komorbiditäten wie Adipositas und Bluthochdruck sind weitere Risikofaktoren für ein progressives atriales Remodeling [51]. In • Abb. 2 wird der Zusammenhang von apnoeassoziierten Arrhythmien und progressivem strukturellen Remodeling veranschaulicht [52].

\section{Elektrophysiologisches Remodeling der Vorhöfe}

Tsai et al. haben diese Umbauprozesse untersucht und dabei nachweislich dehnungsaktivierte Kalziumkanäle festgestellt [53]. Auch Lebek et al. wiesen eine signifikant erhöhte CaMKII-Aktivität bei Patienten mit SBAS im Vergleich zu Patienten ohne SBAS nach [54]. Durch diese entsteht ein signifikanter Repolarisationsgradient, welcher frühe und verspätete Depolarisationen begünstigt und somit kardiale Arrhythmien entstehen lässt $[53,54]$. In einem Tiermodell der OSA führt negativer Trachealdruck zu einer ausgeprägten Verkürzung der atrialen effektiven Refraktärzeit (ERP) und einer erhöhten Induzierbarkeit von VHF, die durch sympathische $\alpha$ - und $\beta$-Wege,
Rhythmuskontrolle bei SBASPatienten mit und ohne CPAPTherapie

Die obstruktive Schlafapnoe und eine niedrige nächtliche Sauerstoffsättigung sind mit einer erhöhten Rezidivrate von VHF nach einer initial erfolgreichen Kardioversion assoziiert [58]. In Beobachtungsstudien wiesen VHF-Patienten, deren OSA mittels CPAP behandelt wurde $(n=39)$, innerhalb der ersten 12 Monate nach elektrischer Kardioversion ein geringeres VHF-Rezidivrisiko auf als Patienten mit unbehandelter OSA (42 versus $82 \% ; P=0,01$ ). Das Rezidivrisiko der VHF-Patienten mit behandelter OSA war ähnlich wie bei Patienten ohne OSA (42 versus $53 \%$ ) [10]. Aktuell wurde erstmals in einer kleinen randomisierten Studie geprüft, ob die Behandlung einer obstruktiven Schlafapnoe mittels CPAP-Therapie das Risiko eines VHF-Rezidivs nach elektrischer Kardioversion senkt (CPAP $n=12$ und Kontrollen $n=13$ ). Hinsichtlich der Rezidivrate $(25 \%)$ und der Zeitdauer bis zum Rezidiv des VHF (129 vs. 109 Tage) zeigte sich im Vergleich der CPAP- mit der Kontrollgruppe kein Unterschied [59]. Jedoch ist die Aussagekraft der Studie zum Beispiel aufgrund der geringen Fallzahlen nicht ausreichend, um einen Effekt der CPAP-Therapie auf das VHF-Rezidivrisiko nach Kardioversion auszuschließen.

In einer Beobachtungsstudie mit 3000 Patienten, die eine Pulmonalvenenisolation erhielten, erlitten $32 \%$ der Gruppe ohne CPAP-Therapie ein VHFRezidiv, während dies bei nur $21 \%$ der Patienten mit effektiver CPAP-Therapie der Fall war $(p=0,003)$ [60]. Bei einer Gruppe von 62 OSA-Patienten, die sich einer Pulmonalvenenisolation unterzogen, ist die CPAP-Therapie im Vergleich zur unbehandelten OSA mit einer höheren VHF-Rezidivfreiheit 12 Monate nach dem Eingriff assoziiert (71,9\% vs. $36,7 \% ; P=0,01)$. Diese Rate ist vergleichbar mit Patienten ohne OSA [61]. Darüber hinaus ist das Risiko eines Versagens der Katheterablationstherapie bei Patienten mit VHF ( $n=174)$ unabhängig mit dem Schweregrad der OSA assoziiert [62]. Die Wahrscheinlichkeit eines 
Rezidivs lag bei Patienten ohne OSA bei $52 \%$, bei Patienten mit schwerer OSA bei $86 \%$ [62, 63]. Zusammenfassend deuten die bisherigen Beobachtungsstudien und elektrophysiologische Studien darauf hin, dass OSA zur Entstehung von VHF und $\mathrm{zu}$ einem VHF-Rezidiv nach elektrischer Kardioversion oder Pulmonalvenenisolation beitragen kann und somit die Effekte antiarrhythmischer Therapiestrategien bei Patienten mit VHF limitiert [16]. Zudem weisen nichtrandomisierte Beobachtungsstudien darauf hin, dass CPAP dazu beitragen kann, den Sinusrhythmus bei Patienten mit VHF, die an OSA leiden, aufrechtzuerhalten [2]. Deshalb wird bereits die klinische Diagnostik und Therapie der OSA zur Vermeidung von VHF-Rezidiven und Verbesserung der Behandlung des VHF empfohlen [2].

Eine Verbesserung der Herzfunktion führt zu einer Abnahme von Apnoen und Hypopnoen [64]. Dieser Zusammenhang ist z.B. bei Patienten nach Herztransplantation [65], in der Frühphase nach Myokardinfarkt [66] sowie unter medikamentöser Therapie der Herzinsuffizienz etabliert $[67,68]$ und gilt auch für die Rhythmisierung von Patienten mit VHF [69]. Beobachtungsstudien zeigen, dass durch eine Kardioversion eine sofortige Reduktion der SBAS aufgrund einer signifikanten Abnahme der zentralen respiratorischen Ereignisse festgestellt werden kann [69]. Beispielsweise sinkt bei einem Kollektiv von Patienten mit VHF und Vorhofflattern $(n=138 ; 86,2 \%$ mit VHF; 13,8\% mit Vorhofflattern) unmittelbar nach der Kardioversion der Apnoe-Hypopnoe-Index (AHI) signifikant $(23,4 \pm 16,3$ vs. $16,3 \pm 11,5 / \mathrm{h} ; p<0,001)$ [69]. Zudem nimmt die Anzahl der CSAPatienten signifikant von 53 auf $23 \mathrm{ab}$ $(p<0,001)$ [69]. Auch der mittels einer Pulmonalvenenisolation hergestellte Sinusrhythmus ist mit einer signifikanten Abnahme des AHI assoziiert ( $p=0,002)$ [70]. Bei Aufrechterhaltung des Sinusrhythmus bleibt der AHI signifikant erniedrigt $(p<0,01)$, jedoch nicht bei $\mathrm{Pa}$ tienten mit VHF-Rezidiv [70].

Das Ziel der laufenden prospektiven Beobachtungsstudie CONSIDER AF (ClinicalTrials.gov: NCT02877745) ist es, Patientengruppen (u.a. Patienten mit
OSA und ZSA) mit einem erhöhten periund postoperativem Risiko nach koronarer Bypassoperation zu identifizieren. Die Ergebnisse dieser Studie können dazu beitragen, das perioperative $\mathrm{Ma}$ nagement von Patienten mit und ohne SBAS zu optimieren, und als Grundlage für künftige Interventionsstudien $\mathrm{zu}$ dienen [71]. In einer groß angelegten Substudie (CONSIDER AF - OBSERVATION: Identification of obstructive sleep apnoea as a novel and potentially treatable risk factor for postoperative de-novo silent and symptomatic atrial fibrillation after elective coronary artery bypass grafting surgery) soll zukünftig der Einfluss von OSA auf das Auftreten von postoperativ neu diagnostiziertem Vorhofflimmern untersucht werden. Die Patienten der Substudie werden dafür postoperativ mittels Langzeit-EKG und einem Smartphone kompatiblen Herzmonitor hinsichtlich des Auftretens von postoperativ neu diagnostiziertem Vorhofflimmern überwacht. Weitere Ergebnisse von randomisierten und größeren Studien, wie z. B. von Traaen et al. [72], sind in den nächsten Jahren zu erwarten. Dies ist die erste randomisierte kontrollierte Studie, die Daten über die Auswirkungen einer CPAP-Therapie bei Patienten mit paroxysmalem VHF und SBAS liefern wird [72].

\section{Effekte der CPAP-Therapie auf nicht rhythmisierende Behandlungsstrategien}

Die nächtliche Behandlung der OSA mit CPAP reduziert sowohl nachts als auch tagsüber die Aktivität des sympathischen Nervensystems [73, 74]. Des Weiteren wurde bei einem Kollektiv von Patienten mit Herzinsuffizienz, reduzierter linksventrikulärer Ejektionsfraktion und OSA gezeigt, dass CPAP akut die linksventrikuläre Wandspannung reduziert [40]. Zudem wurde in einem Großtiermodell der OSA gezeigt, dass es auch einige Stunden nach Beendigung der CPAP-Therapie zu einer Reduktion des linksventrikulären enddiastolischen Volumens und zur Steigerung der Ejektionsfraktion kam [75].

\section{Fazit für die Praxis}

Die Prävalenz von Vorhofflimmern (VHF) ist bei Patienten mit einer schlafbezogenen Atmungsstörung (SBAS) deutlich höher als bei Menschen ohne SBAS. Die zugrunde liegenden Mechanismen sind akut transiente (intermittierende Hypoxie, sympathovagale Aktivierung, intrathorakale Druckschwankungen) sowie chronisch arrhythmogene Mechanismen (atriales Remodeling). Verschiedene nichtrandomisierte Studien beobachten eine geringere VHF-Rezidivrate nach einer initiativ erfolgreichen Rhythmuskontrolle durch die Therapie der SBAS bei diesen Patienten. Deshalb wird die klinische Diagnostik und Therapie der obstruktiven Schlafapnoe (OSA) zur Vermeidung von VHF-Rezidiven und Verbesserung der Behandlung des VHF empfohlen. Dennoch besteht zu diesem Thema noch weiterer Forschungsbedarf in randomisierten Studien. Des Weiteren wird in den meisten Studien nicht zwischen OSA und zentraler Schlafapnoe (ZSA) differenziert, daher treffen die genannten Schlussfolgerungen für ZSA nur eingeschränkt zu.

\section{Korrespondenzadresse}

\section{Victoria Vaas}

Schlafmedizinisches Zentrum der Klinik und Poliklinik für Innere Medizin II, Universitätsklinikum Regensburg Franz-Josef-Strauß-Allee 11, 93053 Regensburg, Deutschland

victoria.vaas@stud.uni-regensburg.de

Funding. Open Access funding enabled and organized by Projekt DEAL.

\section{Einhaltung ethischer Richtlinien}

Interessenkonflikt. V. Vaas, C. Fisser, M. Tafelmeier, D. Linz ${ }^{2}$ und M. Arzt geben an, dass kein Interessenkonflikt besteht.

Für diesen Beitrag wurden von den Autoren keine Studien an Menschen oder Tieren durchgeführt. Für die aufgeführten Studien gelten die jeweils dort angegebenen ethischen Richtlinien.

Open Access. Dieser Artikel wird unter der Creative Commons Namensnennung 4.0 International Lizenz veröffentlicht, welche die Nutzung, Vervielfältigung Bearbeitung, Verbreitung und Wiedergabe in jegli- 
chem Medium und Format erlaubt, sofern Sie den/die ursprünglichen Autor(en) und die Quelle ordnungsgemäß nennen, einen Link zur Creative Commons Lizenz beifügen und angeben, ob Änderungen vorgenommen wurden.

Die in diesem Artikel enthaltenen Bilder und sonstiges Drittmaterial unterliegen ebenfalls der genannten Creative Commons Lizenz, sofern sich aus der Abbildungslegende nichts anderes ergibt. Sofern das betreffende Material nicht unter der genannten Creative Commons Lizenz steht und die betreffende Handlung nicht nach gesetzlichen Vorschriften erlaubt ist, ist für die oben aufgeführten Weiterverwendungen des Materials die Einwilligung des jeweiligen Rechteinhabers einzuholen.

Weitere Details zur Lizenz entnehmen Sie bitte der Lizenzinformation auf http://creativecommons.org/ licenses/by/4.0/deed.de.

\section{Literatur}

1. Schnabel RB, Yin X, Gona P, Larson MG, Beiser AS, McManus DD et al (2015) 50 year trends in atrial fibrillation prevalence, incidence, risk factors, and mortality in the Framingham Heart Study: a cohort study. Lancet 386:154-162. https://doi.org/10. 1016/S0140-6736(14)61774-8

2. Kirchhof P, Benussi S, Kotecha D, Ahlsson A, Atar D, Casadei B et al (2016) 2016 ESC Guidelines for the management of atrial fibrillation developed in collaboration with EACTS. Eur Heart J 37:2893-2962. https://doi.org/10.1093/eurheartj/ehw210

3. Kishore A, Vail A, Majid A, Dawson J, Lees KR, Tyrrell PJ, Smith CJ (2014) Detection of atrial fibrillation after ischemic stroke or transient ischemic attack: a systematic review and metaanalysis. Stroke 45:520-526. https://doi.org/10. 1161/STROKEAHA.113.003433

4. Henriksson KM, Farahmand B, Åsberg S, Edvardsson N, Terént A (2012) Comparison of cardiovascular risk factors and survival in patients with ischemic or hemorrhagic stroke. Int J Stroke 7:276-281. https://doi.org/10.1111/j.1747-4949. 2011.00706.x

5. Grond M, Jauss M, Hamann G, Stark E, Veltkamp R, Nabavi D et al (2013) Improved detection of silent atrial fibrillation using 72-hour Holter ECG in patients with ischemic stroke: a prospective multicenter cohort study. Stroke 44:3357-3364. https://doi.org/10.1161/STROKEAHA.113.001884

6. Kotecha D, Holmes J, Krum H, Altman DG, Manzano L, Cleland JGF et al (2014) Efficacy of $\beta$ blockers in patients with heart failure plus atrial fibrillation: an individual-patient data metaanalysis. Lancet 384:2235-2243. https://doi.org/ 10.1016/S0140-6736(14)61373-8

7. Chun KRJ, Brugada J, Elvan A, Gellér L, Busch $M$, Barrera A et al (2017) The impact of cryoballoon versus radiofrequency ablation for paroxysmal atrial fibrillation on healthcare utilization and costs: an economic analysis from the FIRE AND ICE trial. J Am Heart Assoc. https://doi.org/10.1161/ JAHA.117.006043

8. Lau DH, Nattel S, Kalman JM, Sanders P (2017) Modifiable risk factors and atrial fibrillation. Circulation 136:583-596. https://doi.org/10.1161/ CIRCULATIONAHA.116.023163

9. Heinzer R, Vat S, Marques-Vidal P, Marti-Soler $H$, Andries D, Tobback N et al (2015) Prevalence of sleep-disordered breathing in the general population: the HypnoLaus study. Lancet Respir Med 3:310-318. https://doi.org/10.1016/S22132600(15)00043-0

10. Kanagala R, Murali NS, Friedman PA, Ammash NM, Gersh BJ, Ballman KV et al (2003) Obstructive sleep apnea and the recurrence of atrial fibrillation. Circulation 107:2589-2594. https://doi.org/10. 1161/01.CIR.0000068337.25994.21

11. Gami AS, Pressman G, Caples SM, Kanagala R, Gard JJ, Davison DE et al (2004) Association of atrial fibrillation and obstructive sleep apnea. Circulation 110:364-367. https://doi.org/10.1161/ 01.CIR.0000136587.68725.8E

12. Stevenson $\mathrm{IH}$, Teichtahl $\mathrm{H}$, Cunnington $\mathrm{D}$, Ciavarella S, Gordon I, Kalman JM (2008) Prevalence of sleep disordered breathing in paroxysmal and persistent atrial fibrillation patients with normal left ventricular function. Eur Heart J 29:1662-1669. https://doi.org/10.1093/eurheartj/ehn214

13. Bitter T, Langer C, Vogt J, Lange $M$, Horstkotte $D$, Oldenburg $O$ (2009) Sleep-disordered breathing in patients with atrial fibrillation and normal systolic left ventricular function. Dtsch Arztebl Int 106:164-170. https://doi.org/10.3238/arztebl. 2009.0164

14. Mehra R, Benjamin EJ, Shahar E, Gottlieb DJ, Nawabit R, Kirchner HL et al (2006) Association of nocturnal arrhythmias with sleep-disordered breathing: the Sleep Heart Health Study. Am J Respir Crit Care Med 173:910-916. https://doi.org/ 10.1164/rccm.200509-14420C

15. Leung RST, Huber MA, Rogge T, Maimon N, Chiu K-L, Bradley TD (2005) Association between atrial fibrillation and central sleep apnea. Sleep 28:1543-1546. https://doi.org/10.1093/sleep/28 12.1543

16. Rossi VA, Stradling JR, Kohler M (2013) Effects of obstructive sleep apnoea on heart rhythm. Eur Respir J 41:1439-1451. https://doi.org/10.1183/ 09031936.00128412

17. Li L, Wang Z-W, Li J, Ge X, Guo L-Z, Wang Y et al (2014) Efficacy of catheter ablation of atrial fibrillation in patients with obstructive sleep apnoea with and without continuous positive airway pressure treatment: a meta-analysis of observational studies. Europace 16:1309-1314. https://doi.org/10.1093/europace/euu066

18. Peng Y-J, Yuan G, Ramakrishnan D, Sharma SD, Bosch-Marce M, Kumar GK et al (2006) Heterozygous HIF-1alpha deficiency impairs carotid bodymediated systemic responses and reactive oxygen species generation in mice exposed to intermittent hypoxia. J Physiol (lond ) 577:705-716. https://doi. org/10.1113/jphysiol.2006.114033

19. Daly MD, Scott MJ (1963) The cardiovascular responses to stimulation of the carotid body chemoreceptors in the dog. JPhysiol 165:179-197. https://doi.org/10.1113/jphysiol.1963.sp007051

20. Prabhakar NR, Kumar GK (2004) Oxidative stress in the systemic and cellular responses to intermittent hypoxia. Biol Chem 385:217-221. https://doi.org/ 10.1515/BC.2004.015

21. Peng Y, Yuan G, Overholt JL, Kumar GK, Prabhakar NR (2003) Systemic and cellular responses to intermittent hypoxia: evidence for oxidative stress and mitochondrial dysfunction. Adv Exp Med Biol 536:559-564. https://doi.org/10.1007/978-14419-9280-2 71

22. Chen L, Zhang J, Gan TX, Chen-Izu Y, Hasday JD, Karmazyn $M$ et al (2008) Left ventricular dysfunction and associated cellular injury in rats exposed to chronic intermittent hypoxia. J Appl Physiol 104:218-223. https://doi.org/10.1152/ japplphysiol.00301.2007
23. Liu J-N, Zhang J-X, Lu G, Qiu Y, Di Yang, Yin G-Y, Zhang $X$ (2010) The effect of oxidative stress in myocardial cell injury in mice exposed to chronic intermittent hypoxia. Chin Med J 123:74-78

24. Alonso-Fernández A, García-Río $F$, Arias $M$, Hernanz A, Peña M, Piérola J et al (2009) Effects of CPAP on oxidative stress and nitrate efficiency in sleep apnoea: A randomised trial. Thorax 64:581-586. https://doi.org/10.1136/thx.2008. 100537

25. de Daly MB, Scott MJ (1958) The effects of stimulation of the carotid body chemoreceptors on heart rate in the dog. J Physiol 144:148-166. https://doi.org/10.1113/jphysiol.1958.sp006092

26. Cortelli P, Lombardi C, Montagna P, Parati G (2012) Baroreflex modulation during sleep and in obstructive sleep apnea syndrome. Auton Neurosci 169:7-11. https://doi.org/10.1016/j.autneu.2012. 02.005

27. Guilleminault C, Connolly SJ, Winkle RA (1983) Cardiac arrhythmia and conduction disturbances during sleep in 400 patients with sleep apnea syndrome. Am J Cardiol 52:490-494. https://doi org/10.1016/0002-9149(83)90013-9

28. Shepard JW, Garrison MW, Grither DA, Dolan GF (1985) Relationship of ventricular ectopy to oxyhemoglobin desaturation in patients with obstructive sleep apnea. Chest 88:335-340. https://doi.org/10.1378/chest.88.3.335

29. Koehler U, Fus E, Grimm W, Pankow W, Schäfer H, Stammnitz A, Peter JH (1998) Heart block in patients with obstructive sleep apnoea: pathogenetic factors and effects of treatment. Eur Respir J 11:434-439. https://doi.org/10.1183/ 09031936.98.11020434

30. Zwillich C, Devlin T, White D, Douglas N, Weil J, Martin R (1982) Bradycardia during sleep apnea. Characteristics and mechanism. J Clin Invest 69:1286-1292. https://doi.org/10.1172/jci110568

31. Linz D, Woehrle $H$, Bitter T, Fox $H$, Cowie MR, Böhm M, Oldenburg $O$ (2015) The importance of sleep-disordered breathing in cardiovascular disease. Clin Res Cardiol 104:705-718. https://doi. org/10.1007/s00392-015-0859-7

32. Ferri R, Manconi M, Aricò D, Punjabi NM, Zucconi M (2013) Experimentally induced arousals do not elicit periodic leg motor activity during sleep in normal subjects. Sleep Med 14:85-90. https://doi. org/10.1016/j.sleep.2012.09.021

33. Horner RL, Brooks D, Kozar LF, Tse S, Phillipson EA (1995) Immediate effects of arousal from sleep on cardiac autonomic outflow in the absence of breathing in dogs. J Appl Physiol 79:151-162. https://doi.org/10.1152/jappl.1995.79.1.151

34. Pinto JM, Garpestad E, Weiss JW, Bergau DM, KirbyDA (1993) Hemodynamic changes associated with obstructive sleep apnea followed by arousal in a porcine model. J Appl Physiol 75:1439-1443. https://doi.org/10.1152/jappl.1993.75.4.1439

35. Hedner J, Ejnell H, Sellgren J, Hedner T, Wallin G (1988) Is high and fluctuating muscle nerve sympatheticactivity in the sleep apnoea syndrome of pathogenetic importance for the development of hypertension? J Hypertens 6:S529-S531. https://doi.org/10.1097/00004872-19881204000166

36. Hla KM, Young TB, Bidwell T, Palta M, Skatrud JB, Dempsey J (1994) Sleep apnea and hypertension. A population-based study. Ann Intern Med 120:382-388. https://doi.org/10.7326/00034819-120-5-199403010-00005

37. Xu J, Ding N, Zhang X, Wang N, Sun B, Zhang Ret al (2018) Nocturnal blood pressure fluctuation and associated influential factors in severe obstructive 
sleep apnea patients with hypertension. Sleep Breath 22:1045-1052. https://doi.org/10.1007/ s11325-018-1634-6

38. Somers VK, Dyken ME, Skinner JL (1993) Autonomic and hemodynamic responses and interactions during the Mueller maneuver in humans. J Auton Nerv Syst 44:253-259. https://doi.org/10.1016/ 0165-1838(93)90038-v

39. Linz D, Schotten U, Neuberger H-R, Böhm M, Wirth K (2011) Negative tracheal pressure during obstructive respiratory events promotes atrial fibrillation by vagal activation. Heart Rhythm 8:1436-1443. https://doi.org/10.1016/j.hrthm. 2011.03.053

40. Tkacova R, Rankin F, Fitzgerald FS, Floras JS, Bradley TD (1998) Effects of continuous positive airway pressure on obstructive sleep apnea and left ventricular afterload in patients with heart failure. Circulation 98:2269-2275. https://doi.org/ 10.1161/01.CIR.98.21.2269

41. Fisser C, Götz K, Hetzenecker A, Debl K, Zeman F, Hamer OW et al (2020) Obstructive sleep apnoea but not central sleep apnoea is associated with left ventricular remodelling after acute myocardial infarction. Clin Res Cardiol. https://doi.org/10. 1007/s00392-020-01684-z

42. Chan KH, Wilcox I (2010) Obstructive sleep apnea: novel trigger and potential therapeutic target for cardiac arrhythmias. Expert Rev Cardiovasc Ther 8:981-994.https://doi.org/10.1586/erc.10.80

43. Dimitri H, Ng M, Brooks AG, Kuklik P, Stiles MK, Lau DHet al (2012) Atrial remodeling in obstructive sleep apnea: implications for atrial fibrillation. Heart Rhythm 9:321-327. https://doi.org/10. 1016/j.hrthm.2011.10.017

44. Orban $M$, Bruce $C J$, Pressman GS, Leinveber $P$, Romero-Corral A, Korinek J et al (2008) Dynamic changes of left ventricular performance and left atrial volume induced by the mueller maneuver in healthy young adults and implications for obstructive sleep apnea, atrial fibrillation, and heart failure. Am J Cardiol 102:1557-1561. https:// doi.org/10.1016/j.amjcard.2008.07.050

45. Koshino $Y$, Villarraga HR, Orban $M$, Bruce $C J$, Pressman GS, Leinveber $P$ et al (2010) Changes in left and right ventricular mechanics during the Mueller maneuver in healthy adults: a possible mechanism for abnormal cardiac function in patients with obstructive sleep apnea. Circ Cardiovasc Imaging 3:282-289. https://doi.org/ 10.1161/CIRCIMAGING.109.901561

46. Stöwhas A-C, Namdar M, Biaggi P, Russi EW, Bloch KE, Stradling JR, Kohler M (2011) The effect of simulated obstructive apnea and hypopnea on aortic diameter and BP. Chest 140:675-680. https://doi.org/10.1378/chest.10-2799

47. Kasai T, Bradley TD (2011) Obstructive sleep apnea and heart failure: pathophysiologic and therapeutic implications. J Am Coll Cardio 57:119-127. https://doi.org/10.1016/j.jacc.2010. 08.627

48. Kohler M, Stradling JR (2010) Mechanisms of vascular damage in obstructive sleep apnea. Nat Rev Cardiol 7:677-685. https://doi.org/10.1038/ nrcardio.2010.145

49. Iwasaki Y-K, Kato T, Xiong F, Shi Y-F, NaudP, Maguy A et al (2014) Atrial fibrillation promotion with longterm repetitive obstructive sleep apnea in a rat model. J Am Coll Cardiol 64:2013-2023. https:// doi.org/10.1016/j.jacc.2014.05.077

50. Zhao J, Xu W, Yun F, Zhao H, Li W, Gong Y et al (2014) Chronic obstructive sleep apnea causes atrial remodeling in canines: mechanisms and implications. Basic Res Cardiol 109:427. https://doi. org/10.1007/s00395-014-0427-8

51. Linz D, Linz B, Hohl M, Böhm M (2016) Atrial arrhythmogenesis in obstructive sleep apnea: Therapeuticimplications. SleepMed Rev 26:87-94. https://doi.org/10.1016/j.smrv.2015.03.003

52. Linz D, McEvoy RD, Cowie MR, Somers VK, Nattel S, Lévy $P$ et al (2018) Associations of obstructive sleep apnea with atrial fibrillation and continuous positive airway pressure treatment: a review. JAMA Cardiol 3:532-540. https://doi.org/10.1001/ jamacardio.2018.0095

53. Tsai C-T, Chiang F-T, Tseng C-D, Yu C-C, Wang Y-C, Lai L-P et al (2011) Mechanical stretch of atrial myocyte monolayer decreases sarcoplasmic reticulum calcium adenosine triphosphatase expression and increases susceptibility to repolarization alternans. J Am Coll Cardiol 58:2106-2115. https://doi.org/ 10.1016/j.jacc.2011.07.039

54. Lebek S, Pichler K, Reuthner K, Trum M, Tafelmeier M, Mustroph J, a (2020) Enhanced caMKIIdependent late Ina induces atrial proarrhythmic activity in patients with sleep-disordered breathing. Circ Res 126:603-615. https://doi.org/10. 1161/CIRCRESAHA.119.315755

55. Stevenson IH, Roberts-Thomson KC, Kistler PM, Edwards GA, Spence S, Sanders P, Kalman JM (2010) Atrial electrophysiology is altered by acute hypercapnia but not hypoxemia: implications for promotion of atrial fibrillation in pulmonary disease and sleep apnea. Heart Rhythm 7:1263-1270. https://doi.org/10.1016/j.hrthm.2010.03.020

56. VerrierRL, Dickerson LW (1991) Autonomicnervous system and coronary blood flow changes related to emotional activation and sleep. Circulation 83:II81-1I-89

57. Andreas S, Breska B, Schaumann A, Gonska B, Kreuzer H (1995) Obstructive sleep apnoea and signal averaged electrocardiogram. Eur Respir J 8:546-550

58. Gami AS, Hodge DO, Herges RM, Olson EJ, Nykodym J, Kara T, Somers VK (2007) Obstructive sleep apnea, obesity, and the risk of incident atrial fibrillation. J Am Coll Cardiol 49:565-571. https:// doi.org/10.1016/j.jacc.2006.08.060

59. Caples SM, Mansukhani MP, Friedman PA, Somers VK (2019) The impact of continuous positive airway pressure treatment on the recurrence of atrial fibrillation post cardioversion: A randomized controlled trial. Int J Cardiol 278:133-136. https:// doi.org/10.1016/j.ijcard.2018.11.100

60. Patel D, Mohanty P, Di Biase L, Shaheen M Lewis WR, Quan K et al (2010) Safety and efficacy of pulmonary vein antral isolation in patients with obstructive sleep apnea: the impact of continuous positive airway pressure. Circ Arrhythm Electrophysiol 3:445-451. https://doi org/10.1161/CIRCEP.109.858381

61. Fein AS, Shvilkin A, Shah D, Haffajee Cl, Das S, Kumar K et al (2013) Treatment of obstructive sleep apnea reduces the risk of atrial fibrillation recurrence after catheter ablation. J Am Coll Cardiol 62:300-305. https://doi.org/10.1016/j.jacc.2013. 03.052

62. Matiello $M$, Nadal M, Tamborero D, Berruezo A, Montserrat J, Embid C et al (2010) Low efficacy of atrial fibrillation ablation in severe obstructive sleep apnoea patients. Europace 12:1084-1089. https://doi.org/10.1093/europace/euq128

63. Bitter T, Nölker G, Vogt J, Prinz C, Horstkotte D, Oldenburg $O$ (2012) Predictors of recurrence in patients undergoing cryoballoon ablation for treatment of atrial fibrillation: the independent role of sleep-disordered breathing. J Cardiovasc
Electrophysiol 23:18-25. https://doi.org/10.1111/ j.1540-8167.2011.02148.x

64. Arzt M, Bradley TD (2006) Treatment of sleep apnea in heart failure. Am J Respir Crit Care Med 173:1300-1308. https://doi.org/10.1164/rccm. 200511-1745PP

65. Mansfield DR, Solin P, RoebuckT, Bergin P, Kaye DM, Naughton MT (2003) The effect of successful heart transplant treatment of heart failure on central sleep apnea. Chest 124:1675-1681. https://doi. org/10.1378/chest.124.5.1675

66. Buchner S, Greimel T, Hetzenecker A, Luchner A, Hamer OW, Debl K et al (2012) Natural course of sleep-disordered breathing after acute myocardial infarction. Eur Respir J 40:1173-1179. https://doi. org/10.1183/09031936.00172211

67. Walsh JT, Andrews R, Starling R, Cowley AJ, Johnston ID, Kinnear WJ (1995) Effects of captopril and oxygen on sleep apnoea in patients with mild to moderate congestive cardiac failure. Br Heart J 73:237-241

68. Dark DS, Pingleton SK, Kerby GR, Crabb JE, Gollub SB, Glatter TR, Dunn MI (1987) Breathing pattern abnormalities and arterial oxygen desaturation during sleep in the congestive heart failure syndrome. Improvement following medical therapy. Chest 91:833-836. https://doi.org/10. 1378/chest.91.6.833

69. Fox H, Bitter T, Horstkotte D, Oldenburg O (2016) Cardioversion of atrial fibrillation or atrial flutter into sinus rhythm reduces nocturnal central respiratory events and unmasks obstructive sleep apnoea. Clin Res Cardiol 105:451-459. https://doi. org/10.1007/s00392-015-0940-2

70. Naruse Y, Tada H, Satoh M, Yanagihara M, Tsuneoka H, Hirata Y et al (2012) Radiofrequency catheter ablation of persistent atrial fibrillation decreases a sleep-disordered breathing parameter during a short follow-up period. Circ J 76:2096-2103. https://doi.org/10.1253/circj.CJ-12-0014

71. Tafelmeier M, Knapp M, Lebek S, Floerchinger B, Camboni D, Wittmann Set al (2019) Rationale and design of the CONSIDER AF study. Somnologie 23:17-28. https://doi.org/10.1007/s11818-0190196-6

72. Traaen GM, Aakerøy L, Hunt T-E, Øverland B, Lyseggen E, Aukrust P et al (2018) Treatment of sleep apnea in patients with paroxysmal atrial fibrillation: design and rationale of a randomized controlled trial. Scand Cardiovasc J 52:372-377. https://doi.org/10.1080/14017431.2019.1567933

73. Kohler M, Stoewhas A-C, Ayers L, Senn O, Bloch KE, Russi EW, Stradling JR (2011) Effects of continuous positive airway pressure therapy withdrawal in patients with obstructive sleep apnea: a randomized controlled trial. Am J Respir Crit Care Med 184:1192-1199. https://doi.org/10. 1164/rccm.201106-09640C

74. Kohler M, Pepperell JCT, Casadei B, Craig S, Crosthwaite N, Stradling JR, Davies RJO (2008) CPAP and measures of cardiovascular risk in males with OSAS. Eur Respir J 32:1488-1496. https://doi. org/10.1183/09031936.00026608

75. Genovese J, Huberfeld S, Tarasiuk A, Moskowitz M, Scharf SM (1995) Effects of CPAP on cardiac output in pigs with pacing-induced congestive heart failure. Am J Respir Crit Care Med 152:1847-1853. https://doi.org/10.1164/ajrccm.152.6.8520745 